\title{
Coherent anti-Stokes Raman scattering under electric field stimulation
}

\author{
Erwan Capitaine, ${ }^{1, *}$ Nawel Ould Moussa, ${ }^{1}$ Christophe Louot, ${ }^{1}$ Claire Lefort,,${ }^{1}$ Dominique Pagnoux, ${ }^{1}$ Jean-René Duclère, ${ }^{2}$ \\ Junya F. Kaneyasu, ${ }^{3}$ Hideaki Kano, ${ }^{3,4,5}$ Ludovic Duponchel, ${ }^{6}$ Vincent Couderc, ${ }^{1}$ and Philippe Leproux ${ }^{1, \dagger}$ \\ ${ }^{1}$ XLIM UMR 7252, CNRS-Université de Limoges, 123 avenue Albert Thomas, 87060 Limoges Cedex, France \\ ${ }^{2}$ SPCTS UMR 7315, CNRS-Université de Limoges, 12 rue Atlantis, 87068 Limoges Cedex, France \\ ${ }^{3}$ Department of Applied Physics, Graduate School of Pure and Applied Sciences, University of Tsukuba, \\ 1-1-1 Tennodai, Tsukuba, Ibaraki 305-8573, Japan \\ ${ }^{4}$ Institute of Applied Physics, University of Tsukuba, 1-1-1 Tennodai, Tsukuba, Ibaraki 305-8573, Japan \\ ${ }^{5}$ Tsukuba Research Center for Interdisciplinary Materials Science (TIMS), University of Tsukuba, \\ 1-1-1 Tennodai, Tsukuba, Ibaraki 305-8571, Japan \\ ${ }^{6}$ LASIR UMR 8516, CNRS-Université Lille 1, Sciences et Technologies, 59655 Villeneuve d'Ascq Cedex, France
}

(Received 7 October 2016; revised manuscript received 6 December 2016; published 27 December 2016)

\begin{abstract}
We introduce an experiment using electro-CARS, an electro-optical method based on the combination of ultrabroadband multiplex coherent anti-Stokes Raman scattering (M-CARS) spectroscopy and electric field stimulation. We demonstrate that this method can effectively discriminate the resonant CARS signal from the nonresonant background owing to a phenomenon of molecular orientation in the sample medium. Such molecular orientation is intrinsically related to the induction of an electric dipole moment by the applied static electric field. Evidence of the electro-CARS effect is obtained with a solution of $n$-alkanes $\left(\mathrm{C}_{n} \mathrm{H}_{2 n+2}, 15 \leqslant n \leqslant 40\right)$, for which an enhancement of the CARS signal-to-noise ratio is achieved in the case of $\mathrm{CH}_{2}$ and $\mathrm{CH}_{3}$ symmetric/asymmetric stretching vibrations. Additionally, an electric-field-induced second-harmonic generation experiment is performed in order to corroborate the orientational organization of molecules due to the electric field excitation. Finally, we use a simple mathematical approach to compare the vibrational information extracted from electro-CARS measurements with spontaneous Raman data and to highlight the impact of electric stimulation on the vibrational signal.
\end{abstract}

DOI: 10.1103/PhysRevB.94.245136

\section{INTRODUCTION}

For more than a decade, coherent anti-Stokes Raman scattering (CARS) [1-3] has received more and more interest, and it is now one of the most efficient tools in the field of microspectroscopy applied to biological systems. This ongoing interest is related to several advantages, among which is the label-free ability of the method based on the inherent vibrational modes of the sample molecules. CARS requires a third-order nonlinear process to take place in the sample. To do so, a pump wave, a Stokes wave, and a probe wave at $\omega_{p}, \omega_{s}$, and $\omega_{p r}$ frequencies are simultaneously launched in the sample. When the frequency difference between $\omega_{p}$ and $\omega_{s}$ matches the frequency of the vibrational modes, a resonance phenomenon occurs. The probe beam is then used to produce an anti-Stokes wave at $\omega_{a s}$ frequency, with $\omega_{a s}=\omega_{p r}+\left(\omega_{p}-\omega_{s}\right)$. The directionality of the latter, due to the phase-matching condition between the four waves, permits its collection to be done easily [4]. Additionally, the detection of such an anti-Stokes signal is not affected by the one-photon fluorescence contribution. As a nonlinear process, CARS is obtained in tight-focusing conditions and thus represents a confocal microscopy method with intrinsic sectioning capability [3]. Moreover, the use of a broadband Stokes wave enables the acquisition of a full CARS spectrum in only one measurement, with this configuration being known as multiplex CARS (M-CARS) [5-7]. However, a nonresonant background (NRB) remains a major drawback with CARS. It

\footnotetext{
*erwan.capitaine@xlim.fr

†philippe.leproux@xlim.fr
}

occurs because among all the quantum pathways that can be followed by the system to contribute to the signal at the $\omega_{a s}$ frequency, some do not involve a resonance with a vibrational state. This nonresonant contribution interferes with the resonant part of the signal and causes the distinctive distortions of CARS spectra in comparison with spontaneous Raman spectra. This phenomenon can explain why, so far, CARS has not been established as a widespread microspectroscopy technique. Nevertheless, several methods have been developed to reduce or suppress the NRB. Epidetected CARS (E-CARS) was proposed for its insensitivity to the NRB of the sample solvent [2]. Polarization-sensitive CARS (P-CARS) was designed to discriminate the resonant and nonresonant contributions by their polarization vector directions [8]. Regrettably, these two techniques suffer from the global reduction of the anti-Stokes response [9]. Other techniques were introduced, such as time-resolved CARS [10], temporal or spectral interferometry CARS [11,12], and frequency-modulated CARS [13], but they involve some complexity in the setup in terms of both optics and electronics.

A particular property of the CARS process is now introduced. It has been shown that the anti-Stokes signal intensity of a specific bond varies as $\langle\cos \psi\rangle^{6}$, with $\psi$ being the angle between the optical electromagnetic field polarization vectors and the molecular bond directions [14]. Therefore, the CARS technique is based on the assumption that there are enough chemical bonds aligned with the polarization vectors of the incident beams. Nonetheless, while in a solution the molecular agitation guarantees that some bonds are adequately aligned during the probing duration, that is not necessarily the case for single lipid bilayers [15], multilamellar phospholipid onions [16], or multilamellar vesicles $[17,18]$. For such samples, a 
specific orientation of the molecules composing the membrane or in contact with it has been demonstrated which can cause the resonant contribution to be overwhelmed by the nonresonant one. To increase the resonant signal, one would have to locally control the orientation of the molecules, as can be done with liquid crystals under the influence of a continuous electric field [19].

In previous works, we have demonstrated the application of subnanosecond M-CARS spectroscopy to the molecular fingerprinting of living cells [20] and to the study of molecular chirality by means of the CARS Raman optical activity (CARS-ROA) technique [21]. In this paper we introduce the efficient combination of ultrabroadband M-CARS spectroscopy and electric field stimulation. We show that this electro-optical process, which we call electro-CARS, can discriminate the resonant signal from the NRB by means of molecular orientation and thus that it can be applied to the enhancement of the CARS signal-to-noise (SN) ratio. This demonstration of electro-CARS is realized using a solution of $n$-alkanes. Additionally, an electric-field-induced secondharmonic generation (EFISHG) experiment is performed in order to prove the orientational organization of molecules due to the electric field excitation. Finally, a simple mathematical approach is introduced to compare the vibrational information extracted from electro-CARS measurements with spontaneous Raman data and to put in evidence the impact of electric stimulation on the vibrational signal.

\section{EXPERIMENTAL SETUP}

The electro-CARS experimental setup is depicted in Fig. 1. A subnanosecond microchip Nd:YAG laser (Hamamatsu L11435, $1064 \mathrm{~nm}, 400 \mathrm{ps}, 25 \mathrm{kHz}, 500 \mathrm{~mW}$, linearly polarized) is used to generate both the pump and probe radiations

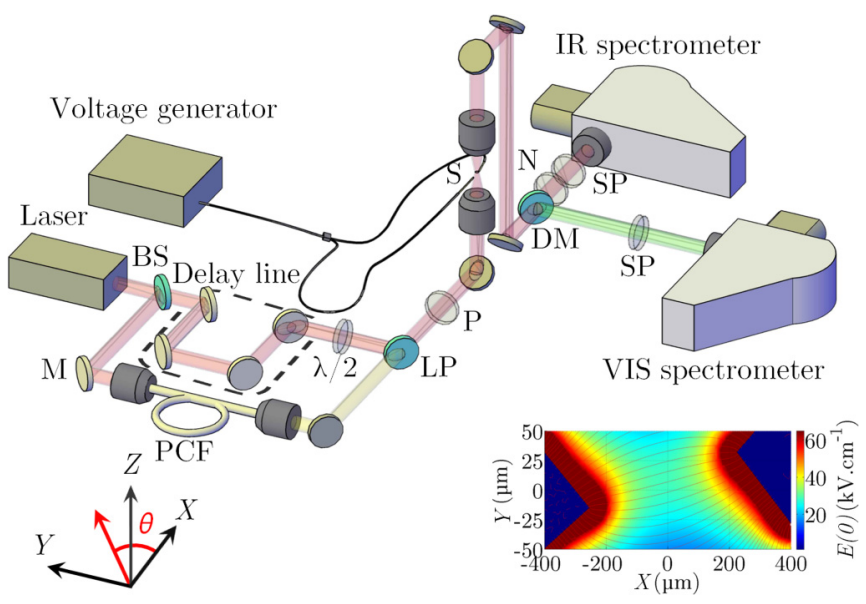

FIG. 1. Simplified schematic of the experimental setup for electro-CARS and EFISHG (BS: beam splitter; DM: dichroic mirror; LP: long-pass filter; $\mathrm{M}$ : mirror; $\mathrm{N}$ : notch filter; $\mathrm{P}$ : polarizer; $\mathrm{PCF}$ : photonic crystal fiber; $S$ : sample; SP: short-pass filter; $\lambda / 2$ : half-wave plate). $\theta$ is defined as the angle between the microscope stage $X$ axis and the optical polarization vectors. Inset: Computed cartography of the electric field between the two electrodes. In the center of the sample, the angle between the $X$ axis and the field lines is about $4^{\circ}$; the average pump and Stokes powers are 30 and $6 \mathrm{~mW}$, respectively. $\left(\omega_{p}=\omega_{p r}\right)$. A part of the beam is launched into a photonic crystal fiber (PCF) to produce an infrared supercontinuum Stokes wave. A delay line is set on the pump path to ensure the temporal overlapping with the Stokes beam, followed by a half-wave plate to control the pump polarization direction. The two beams are then spatially recombined by means of a 1064-nm long-pass edge filter. Because of the supercontinuum radiation coming from the $\mathrm{PCF}$ being randomly polarized, a polarizer is inserted on the combined path to have the pump and Stokes polarization vectors collinear to each other. One microscope objective [Nikon x60, water immersion, numerical aperture (NA) $=1.20$ ] is used to focus both beams into the sample. A second one (Nikon $\mathrm{x} 40, \mathrm{NA}=0.60$ ) permits us to collect the signal coming from the sample in the forward direction. The lateral and axial resolutions are 0.5 and $1.0 \mu \mathrm{m}$, respectively, for a sample thickness of $75.0 \pm 0.3 \mu \mathrm{m}$. To control the $z$ position of the focusing volume in the sample, a piezo-electric stage (Thorlabs MZS500-E) with 25-nm resolution is used. A $685-\mathrm{nm}$ dichroic mirror allows separating near-infrared wavelengths (CARS signal) from visible ones [second harmonic $(\mathrm{SH})$ at $532 \mathrm{~nm}$ ]. On the infrared detection path, the remaining pump is removed by a 1064-nm notch filter and a 1050-nm short-pass filter. The near-infrared light can then be directed to a spectrometer (Lucir Z3208i-HK) combined with a CCD camera (Andor iVac 316). On the visible detection path, the spectrometer (Princeton Instruments LS785) and CCD camera (Princeton Instruments PIXIS 100 BRDD) are preceded by a $650-\mathrm{nm}$ short-pass filter to avoid the occurrence of SHG artifacts. To implement the electric stimulation, two silver tip-shaped, flat electrodes separated by approximatively $500 \mu \mathrm{m}$ and linked to a continuous voltage generator are brought to the sample encapsulated between two coverslips. The potential difference between the electrodes then produces a static electric field of amplitude $E(0)$. The sample model of our study is a solution of $n$-alkanes $\left(\mathrm{C}_{n} \mathrm{H}_{2 n+2}, 15 \leqslant n \leqslant 40\right)$. As they are nonpolar molecules (dipole moment $\mu=0.063 \mathrm{D}$ ) [22], $n$-alkanes are a relevant model for proving the ability of our method to analyze a wide range of objects.

\section{RESULTS}

\section{A. M-CARS spectroscopy under electric field stimulation}

We first investigated the evolution of the vibrational modes' signal intensity as a function of the electric field amplitude and according to two different relative orientations between the optical and electric fields. The first (collinear) and second (perpendicular) configurations correspond, respectively, to $0^{\circ}$ and $90^{\circ}$ angles between the optical field polarization vectors and the direction of the electric field, given by the orientation of the electrodes. Figure 2(a) plots the CARS spectrum of the alkane solution in the collinear configuration for nine different values of $E(0)$, first from 0 to $18 \mathrm{kV} \mathrm{cm}^{-1}$ with a step of $6 \mathrm{kV} \mathrm{cm}{ }^{-1}$ and then from 18 to $33 \mathrm{kV} \mathrm{cm}^{-1}$ with a step of $3 \mathrm{kV} \mathrm{cm}^{-1}$. In the $\mathrm{CH}$ stretching region (3000 to $2700 \mathrm{~cm}^{-1}$ ), a clear increase in the CARS signal intensity can be observed when the electric field is applied and intensified. On the contrary, the electric excitation causes the signal to decrease in the fingerprint region (1500 to $1200 \mathrm{~cm}^{-1}$ ), but 
(a)

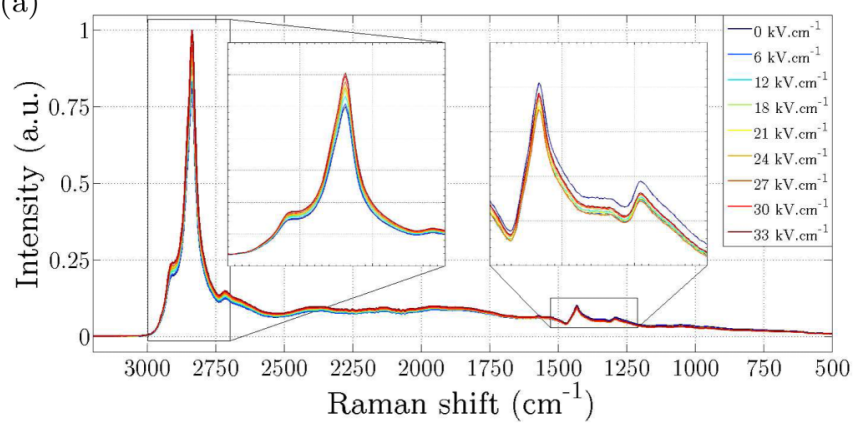

(b)

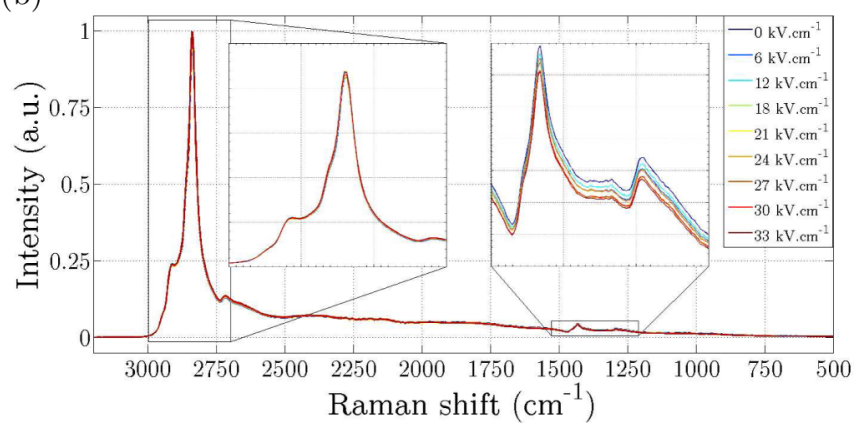

FIG. 2. CARS spectra of an $n$-alkane solution under the influence of a static electric field whose amplitude $E(0)$ varies from 0 to $33 \mathrm{kV} \mathrm{cm}^{-1}$ (blue to red) for the (a) collinear and (b) perpendicular configurations. Insets: Close-ups of the $\mathrm{CH}$ stretching and fingerprint regions. The CCD integration time is $600 \mathrm{~ms}$.

this decrease does not vary monotonically with $E(0)$. In the perpendicular configuration [Fig. 2(b)], the evolution of the CARS signal intensity with the electric field appears to be weak in the $\mathrm{CH}$ stretching region, whereas it corresponds to an obvious decrease in the fingerprint region.

To better analyze the evolution of the CARS signal with the electric field, the previous sets of spectra have been replotted by normalizing the data with respect to the CARS spectrum measured at $E(0)=0 \mathrm{kV} \mathrm{cm}{ }^{-1}$ in both the collinear [Fig. 3(a)] and perpendicular [Fig. 3(b)] configurations. In the collinear case, a gradual increase in the signal intensity is clearly seen in the $\mathrm{CH}$ stretching region, with about $22 \%$ growth at $2840 \mathrm{~cm}^{-1}$. In the perpendicular case, two features corresponding to Raman shifts of 2925 and $2840 \mathrm{~cm}^{-1}$ diverge from the overall behavior by presenting distinct drops. For these peaks, which correspond, respectively, to $\mathrm{CH}_{3}$ and $\mathrm{CH}_{2}$ symmetric stretching, a strictly opposite evolution is obtained between the collinear and perpendicular configurations. This opposite behavior is distinctly revealed by Fig. 3(c), in which the normalized CARS spectra acquired in both configurations for $E(0)=33 \mathrm{kV} \mathrm{cm}^{-1}$ are compared. In addition, the same behavior appears for $\mathrm{CH}_{3}$ and $\mathrm{CH}_{2}$ asymmetric stretching (2960 and $2890 \mathrm{~cm}^{-1}$ ). Regarding the fingerprint region, the signal decrease remains around $4 \%$ for the collinear case whatever the value of $E(0)$, whereas it progressively reaches almost $8 \%$ for the perpendicular case. Moreover, the variation in the signal intensity $(<1.5 \%)$ from 3200 to $3000 \mathrm{~cm}^{-1}$ is weak compared to the variation of the CARS signal in the $\mathrm{CH}$ stretching region. This means that the electric excitation has a lower impact on the NRB than on the vibrational signal. (a)

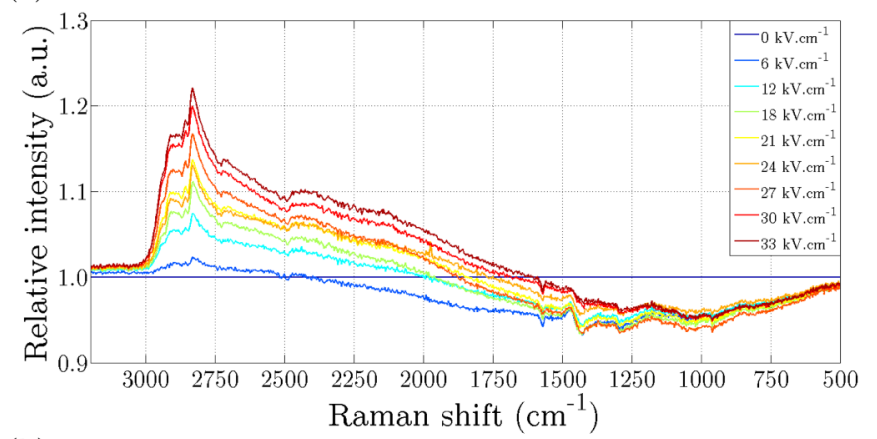

(b)

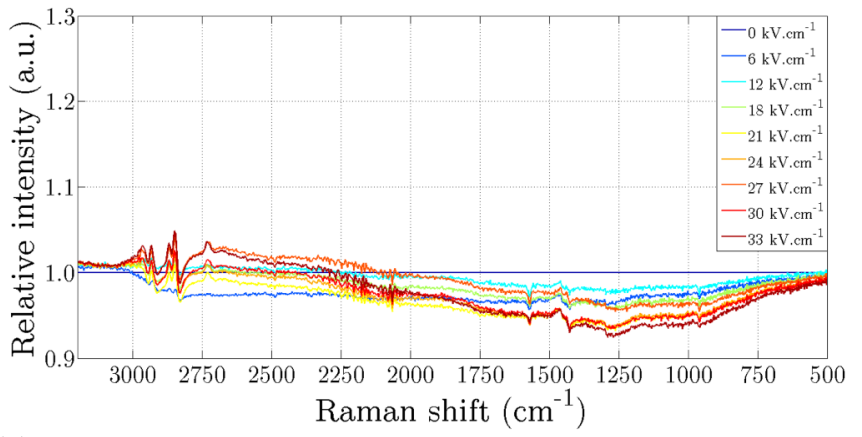

(c)

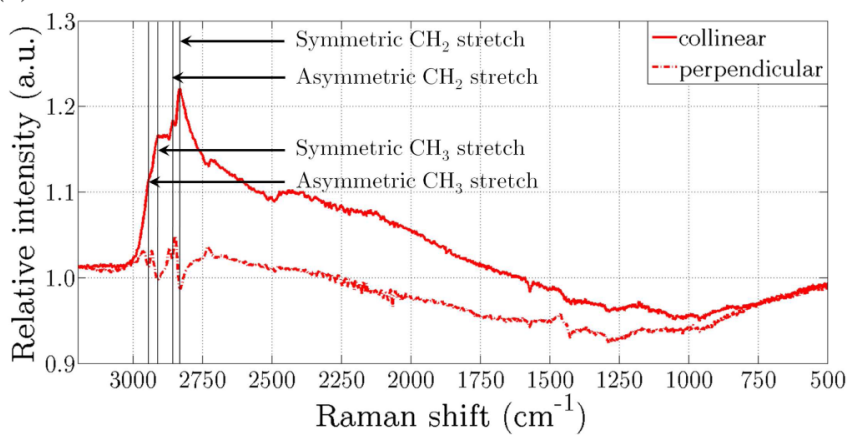

FIG. 3. CARS spectra of the $n$-alkane solution normalized with respect to the spectrum measured for $E(0)=0 \mathrm{kV} \mathrm{cm}^{-1}$ (blue to red) for the (a) collinear and (b) perpendicular configurations. (c) Comparison between both configurations for $E(0)=33 \mathrm{kV} \mathrm{cm}^{-1}$.

This differential effect is particularly strong in the collinear configuration. Therefore, following our observations, when a static electric field is applied to the sample, the intensity of the CARS signal containing the vibrational information varies according to the angle between the electric field and the optical polarization. The origin of this phenomenon would thus be a reorientation of the chemical bonds. As the signal intensity of $\mathrm{CH}$ stretching increases in the collinear case and conversely decreases in the perpendicular one, one can suppose that the $n$-alkane molecules preferentially align their $\mathrm{CH}_{3}$ and $\mathrm{CH}_{2}$ bonds in the electric field direction. However, because $n$-alkanes are nonpolar molecules, it is expected that the permanent dipole moment cannot drive their orientation in the electric field. Subsequently, we investigated the EFISHG response of the $n$-alkane solution in order to clarify the possible contribution of an induced dipole moment to the molecular orientation process, taking into account the dependence of the SH signal on the dipole moment [23]. 


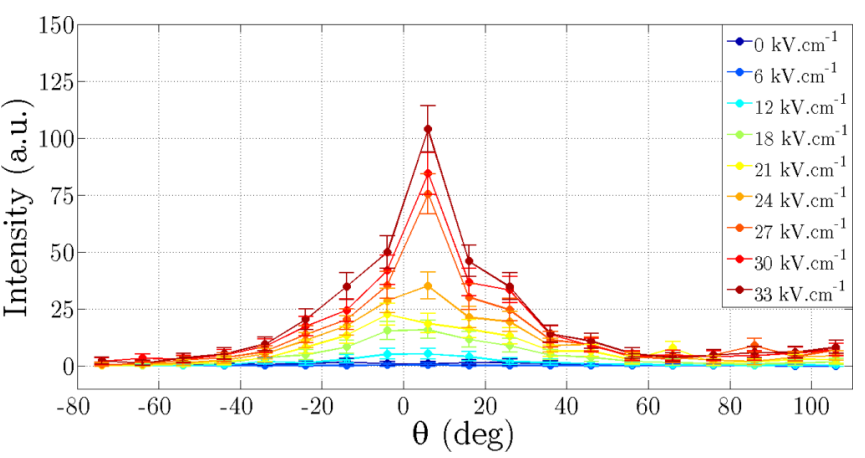

FIG. 4. Intensity of the EFISHG signal as a function of $E(0)$ and $\theta$, the angle between the microscope stage $X$ axis and the optical pump polarization vector. The CCD integration time is $600 \mathrm{~ms}$.

\section{B. Supplementary EFISHG measurement}

A typical EFISHG measurement consists of the application of a static electric field $\vec{E}(0)$ and a fundamental optical field $\vec{E}(\omega)$ that will give rise to an induced polarization $\vec{P}(2 \omega)$ in a liquid or a molecular solution, thus producing a SH optical field $\vec{E}(2 \omega)$ [24]. We investigated EFISHG in the $n$-alkane sample excited by the 1064-nm optical pump radiation. The intensity of the SH signal at $532 \mathrm{~nm}$ was measured as a function of the electric field amplitude and of the angle $\theta$ between the $X$ axis and the pump polarization vector. $\theta$ was varied by rotating the half-wave plate and the polarizer. Figure 4 depicts the obtained SH intensity as a function of $E(0)$ and $\theta$. When no electric field is applied, no SH signal is detected. However, the intensity at $532 \mathrm{~nm}$ appears and increases significantly for $E(0)>12 \mathrm{kV}$ $\mathrm{cm}^{-1}$, revealing at the same time the existence of an optimum value of $\theta$, for which the $\mathrm{SH}$ intensity is maximum. This value is $\theta=5^{\circ}$, and it matches pretty well the actual orientation of the electric field lines between the electrodes (about $4^{\circ}$ ). At this point, we can assert that the EFISHG is the most effective when the optical beam polarization vector is aligned with the static electric field.

\section{DISCUSSION}

\section{A. Molecular orientation process}

We discuss here the contribution of the $n$-alkane dipole moment to the supposed molecular orientation process, following our observations related to EFISHG. For this purpose, it is necessary to look at the relationship between the induced SH signal and the dipole moment. For a single molecule, the induced polarization is [25]

$$
p_{i}(2 \omega)=E_{j}(\omega) E_{k}(\omega)\left[\beta_{i j k}+\left(\gamma_{i j k l}^{e}+\gamma_{i j k l}^{\nu}\right) E_{l}(0)\right],
$$

where $\beta_{i j k}$ is a component of the second-order polarizability tensor and $\gamma_{i j k l}^{e}$ and $\gamma_{i j k l}^{v}$ are components of the purely electronic and vibrational third-order polarizability tensors, respectively. The mean single-molecule polarization is then obtained by averaging $p_{i}(2 \omega)$ for all the possible orientations of molecules in the liquid:

$$
\langle p(2 \omega)\rangle=\gamma_{X X X X} E(\omega)^{2} E(0)\langle\cos \phi\rangle^{2} .
$$

Equation (2) introduces the average third-order polarizability $\gamma_{X X X X}$ and the reference axis along which the static electric field is aligned $X ;\langle\cos \phi\rangle$ is the degree of orientation, with $\phi$ being the angle between the chemical bond and the fundamental optical field polarization vector orientation. In the case of a static electric field, the average third-order polarizability can be decomposed into three parts:

$$
\gamma_{X X X X}=\gamma^{e}+\gamma^{v}+\gamma^{r} .
$$

Here, $\gamma^{e}$ represents the average electronic contribution, $\gamma^{\nu}$ is the average vibrational contribution, and $\gamma^{r}$ is the average dipolar rotational contribution. The latter depends on the dipole moment as

$$
\gamma^{r}=\frac{\vec{\beta} \cdot \vec{\mu}}{5 k_{B} T}
$$

with $\vec{\beta}$ being the vector part of the second-order polarizability tensor, $\vec{\mu}$ being the dipole moment of the molecule, $k_{B}$ being the Boltzmann constant, and $T$ being the temperature.

Moreover, the macroscopic polarization can be obtained from the mean single-molecule polarization as follows:

$$
P(2 \omega)=N f_{0} f_{\omega}^{2} f_{2 \omega}\langle p(2 \omega)\rangle,
$$

where $N$ is the number density of molecules in the sample and $f_{0}, f_{\omega}$, and $f_{2 \omega}$ are the local field factors due to, respectively, the static electric field, the fundamental optical field, and the $\mathrm{SH}$ one. If we admit that $\vec{\beta}$ is collinear to the optical field polarization vector, then $\vec{\beta} \cdot \vec{\mu}=\beta \mu\langle\cos \phi\rangle$, and we get from (2) and (5)

$$
I(2 \omega) \propto\left\{E(\omega)^{2} E(0)\langle\cos \phi\rangle^{2}\left[\left(\gamma^{e}+\gamma^{\nu}\right)+\frac{\beta \mu}{5 k_{B} T}\langle\cos \phi\rangle\right]\right\}^{2} .
$$

From this last expression we find $I(2 \omega)$ is maximum for $\phi=0^{\circ}$ and zero for $\phi= \pm 90^{\circ}$. This behavior is in good agreement with the experimental results of Fig. 4 when taking into account that $\theta-\phi=5^{\circ}$ given the tilt of electric field lines between the electrodes (see Fig. 1). In that case, it can be asserted that the induced dipole moment of the molecule is aligned with the electric field, and consequently, this supports the idea of a molecular preferential orientation.

More specifically, a hypothesis would be a change in the electron density distribution for some $\mathrm{CH}_{2}$ and $\mathrm{CH}_{3}$ bonds. As represented in Fig. 5, the hydrogen and carbon atoms, as well as the carbon-hydrogen bonds, would undergo a distortion of their electron cloud in the opposite direction of the applied electric field. This modification would create a counter electric field opposed to the applied one and would generate an induced electric dipole moment. The latter would then align according to the electric field. As a result, the average direction defined by the $\mathrm{CC}$ backbone of the molecules would lie in a plane perpendicular to the applied electric field, while the $\mathrm{CH}$ bonds would tend to be aligned with the field. Such a hypothesis is consistent with the observations made in the M-CARS spectroscopy experiment, where the signal intensity of the $\mathrm{CH}$ stretching vibrations increases (decreases) in the collinear (perpendicular) case. Furthermore, a rotation of $\mathrm{CH}_{2}$ and $\mathrm{CH}_{3}$ bonds could be driven by the induced dipole moment in the presence of a strong electric field since the single covalent CC bonds of the backbone do not prevent such a rotation. Also, other physical quantities could be affected by 
(a)

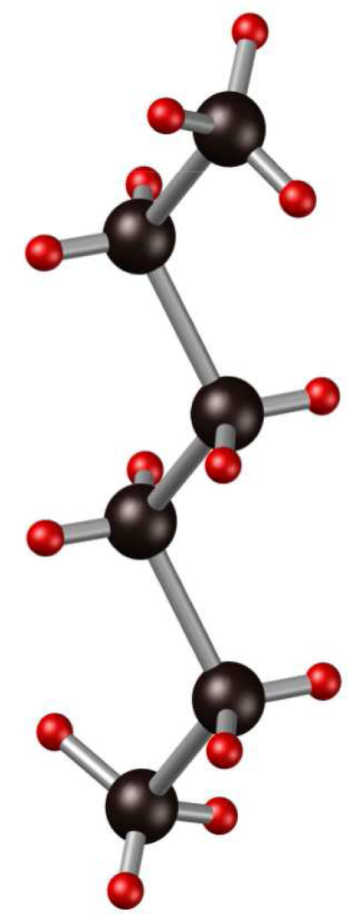

(b)

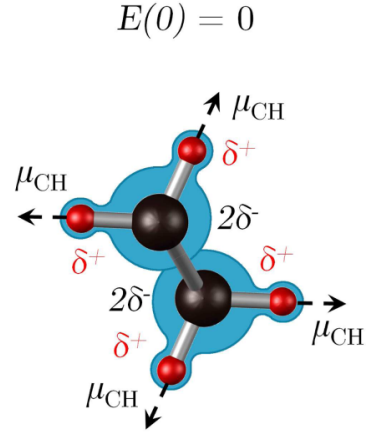

(c)

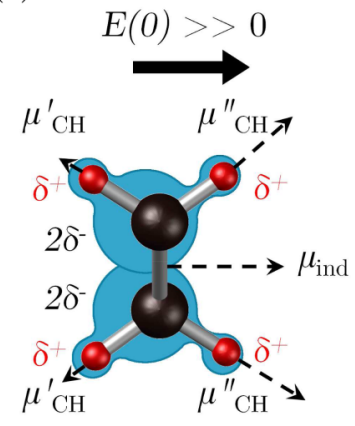

FIG. 5. (a) Schematic representation of an $n$-alkane molecule. Simplified representation of two consecutive $\mathrm{CH}_{2}$ bonds: (b) without and (c) with the influence of a static electric field. The carbon and hydrogen atoms are, respectively, represented in black and red, with their partial charges being $2 \delta^{-}$and $\delta^{+}$. The electron density distribution is represented in blue.

the electric stimulation, such as the components of the secondand third-order polarizability tensors $\beta$ and $\boldsymbol{\gamma}$, which are involved in the chemical bond orientation. The macroscopic quantities $\chi^{(2)}$, linked to $\vec{\mu}$ and $\boldsymbol{\beta}$, and $\chi^{(3)}$, linked to $\boldsymbol{\gamma}$, would then be correspondingly affected by the application of the static electric field.

\section{B. Impact on the vibrational signal}

The impact of electric field stimulation on the vibrational signal is now addressed with the support of a simple mathematical approach. Figure 6 plots four different representations of the vibrational information, namely, the spontaneous Raman spectrum (solid black line), the raw M-CARS spectrum $[S(\omega, E(0))$, dashed black line], the partial derivative of the M-CARS spectrum with respect to $\omega(\delta S / \delta \omega$, blue line $)$, and the mixed partial derivative of the M-CARS spectrum with respect to $\omega$ and $E(0)\left[\delta^{2} S / \delta E(0) \delta \omega\right.$, red line].

Regarding the partial derivative $\delta S / \delta \omega$, it is anticipated that the vibrational information would be contained by the positive peaks of the function, given the well-known mixing of resonant and nonresonant signals in the raw CARS spectrum [26]. This expectation is clearly verified in Fig. 6 by comparing $\delta S / \delta \omega$ with the Raman spectrum in both the $\mathrm{CH}$ stretching and fingerprint ( $\mathrm{CH}$ bending/twisting) regions (see the caption of Fig. 6 for the complete list of revealed vibrations). From there, the impact of the electric field on the vibrational signal

(a)

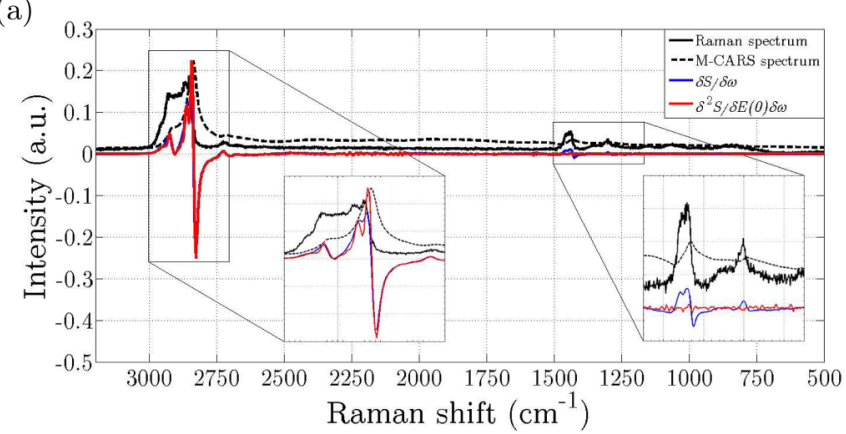

(b)

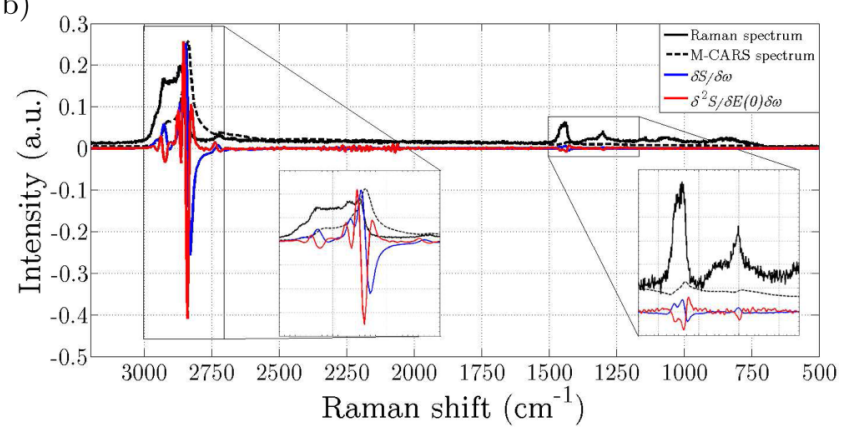

FIG. 6. Comparison of four different representations of the vibrational information: spontaneous Raman spectrum (solid black line), raw M-CARS spectrum [ $S(\omega, E(0))$, dashed black line], partial derivative of the M-CARS spectrum with respect to $\omega(\delta S / \delta \omega$, blue line), and the mixed partial derivative of the M-CARS spectrum with respect to $\omega$ and $E(0)\left[\delta^{2} S / \delta E(0) \delta \omega\right.$, red line $]$ for (a) the collinear and (b) the perpendicular configurations. The following molecular vibrations appear in the figure: $\mathrm{CH}_{3}$ asymmetric/symmetric stretching (2960, $\left.2890 \mathrm{~cm}^{-1}\right), \mathrm{CH}_{2}$ asymmetric/symmetric stretching (2925, $\left.2840 \mathrm{~cm}^{-1}\right)$, and $\mathrm{CH}_{2}$ vibrational bending (1460-1440 $\left.\mathrm{cm}^{-1}\right)$ and twisting $\left(1300 \mathrm{~cm}^{-1}\right)$.

can be easily grasped by examining the $\delta^{2} S / \delta E(0) \delta \omega$ curve. In the collinear configuration [Fig. 6(a)], it is obvious that the blue and red curves show similar in-phase variations in the $\mathrm{CH}$ stretching region. This confirms that the electric stimulation results in strengthening the molecular vibrations in this case, leading to a higher $\mathrm{SN}$ ratio. In the perpendicular configuration [Fig. 6(b)], the opposite behavior is observed, with a noticeable phase opposition between the blue and red curves. The lowering of the vibrational signal takes place in the fingerprint region as well, where in contrast, no distinct effect of the electric field could be seen for the collinear case. We assign this last assessment to the more complex behavior of the bending (1460-1440 $\mathrm{cm}^{-1}$ ) and twisting $\left(1300 \mathrm{~cm}^{-1}\right)$ modes in comparison to the stretching ones.

\section{CONCLUSION}

In summary, we demonstrated an experiment for the socalled electro-CARS spectroscopy based on the combination of M-CARS and electric excitation of the sample. This electrooptical method allows us to easily investigate molecular vibrations over an ultrabroad spectral range and under the influence of an electric field. We showed in a solution of $n$-alkanes 
that electro-CARS can differentiate the resonant signal from the NRB by means of an electric-field-induced modification of the molecular orientation. This process is corroborated by a supplementary EFISHG measurement that elucidates the contribution of the induced dipole moment. Finally, we introduced a simple mathematical approach for extracting the vibrational information from electro-CARS spectra and for highlighting the impact of electric stimulation on the vibrational signal.

We have proved that our method could increase the number of chemical bonds orientated in the direction of the optical field polarization vectors, i.e., the number of oscillators that contribute to the signal. So it allows us to improve the SN ratio and the sensitivity of classical M-CARS spectroscopy. To a greater extent electro-CARS spectroscopy represents a tool to explore and/or control the physicochemical characteristics and processes in a wide range of samples, especially fluids, with weak or strong dipole moment. The impact of the method is foreseen in physics, chemistry, biology, and even medicine, where a direct application of electro-CARS is, for example, anticancer electrochemotherapy [27]. In this case, electro-CARS based on nanosecond or picosecond electric pulses [28] would permit us to investigate the cell membrane modifications and monitor the drug delivery process without damaging the sample.

\section{ACKNOWLEDGMENTS}

We wish to acknowledge the support of the French defense agency (Direction Générale de l'Armement) and by funds from ANR through the NEOSPRAM project (ANR-14-ASTR0014).
[1] A. Zumbusch, G. R. Holtom, and X. S. Xie, Phys. Rev. Lett. 82, 4142 (1999).

[2] J.-X. Cheng, A. Volkmer, L. D. Book, and X. S. Xie, J. Phys. Chem. B 105, 1277 (2001).

[3] A. Volkmer, J.-X. Cheng, and X. S. Xie, Phys. Rev. Lett. 87, 023901 (2001).

[4] J.-X. Cheng and X. S. Xie, J. Phys. Chem. B 108, 827 (2004).

[5] M. Okuno, H. Kano, P. Leproux, V. Couderc, and H. Hamaguchi, Opt. Lett. 33, 923 (2008).

[6] M. Müller and J. M. Schins, J. Phys. Chem. B 106, 3715 (2002).

[7] G. W. H. Wurpel, J. M. Schins, and M. Müller, Opt. Lett. 27, 1093 (2002).

[8] J.-X. Cheng, L. D. Book, and X. S. Xie, Opt. Lett. 26, 1341 (2001).

[9] C. Freudiger and X. S. Xie, in Coherent Raman Scattering Microscopy, edited by J.-X. Cheng and X. S. Xie (CRC Press, Boca Raton, FL, 2013), Chap. 4, pp. 100-113.

[10] A. Volkmer, L. D. Book, and X. S. Xie, Appl. Phys. Lett. 80, 1505 (2002).

[11] C. Vinegoni, J. S. Bredfeldt, and D. L. Marks, Opt. Express 12, 331 (2004)

[12] C. L. Evans, E. O. Potma, and X. S. Xie, Opt. Lett. 29, 2923 (2004).

[13] F. Ganikhanov, C. L. Evans, B. G. Saar, and X. S. Xie, Opt. Lett. 31, 1872 (2006)

[14] A. V. Kachynski, A. N. Kuzmin, P. N. Prasad, and I. I. Smalyukh, Opt. Express 16, 10617 (2008).

[15] E. O. Potma and X. S. Xie, J. Raman Spectrosc. 34, 642 (2003).
[16] J.-X. Cheng, S. Pautot, D. A. Weitz, and X. S. Xie, Proc. Natl. Acad. Sci. USA 100, 9826 (2003).

[17] G. W. H. Wurpel, H. A. Rinia, and M. Müller, J. Microsc. (Oxford, UK) 218, 37 (2004).

[18] J. Duboisset, P. Berto, P. Gasecka, F.-Z. Bioud, P. Ferrand, H. Rigneault, and S. Brasselet, J. Phys. Chem. B 119, 3242 (2015).

[19] B. G. Saar, H.-S. Park, X. S. Xie, and O. D. Lavrentovich, Opt. Express 15, 13585 (2007).

[20] M. Okuno, H. Kano, P. Leproux, V. Couderc, J. Day, M. Bonn, and H. Hamaguchi, Angew. Chem., Int. Ed. 49, 6773 (2010).

[21] K. Hiramatsu, M. Okuno, H. Kano, P. Leproux, V. Couderc, and H. Hamaguchi, Phys. Rev. Lett. 109, 083901 (2012).

[22] U. Stumper, in Molecular Motions in Liquids: Proceedings of the 24th Annual Meeting of the Société de Chimie Physique, Paris-Orsay, 2-6 July 1972 (Springer, Dordrecht, 1974), pp. 655-660.

[23] Y. Shimbo, E. Gorecka, D. Pociecha, F. Araoka, M. Goto, Y. Takanishi, K. Ishikawa, J. Mieczkowski, K. Gomola, and H. Takezoe, Phys. Rev. Lett. 97, 113901 (2006).

[24] B. F. Levine and C. G. Bethea, Appl. Phys. Lett. 24, 445 (1974).

[25] G. He and S. H. Liu, in Physics of Nonlinear Optics (World Scientific, Singapore, 1999), Chap. 4, p. 86.

[26] C. Fang, F. Lu, W. Zheng, and Z. Huang, Opt. Express 18, 15714 (2010).

[27] C. Y. Calvet and L. M. Mir, Cancer Metastasis Rev. 35, 165 (2016).

[28] D. Arnaud-Cormos, R. P. O'Connor, P. Leveque, and V. Couderc, IEEE Photon. Technol. Lett. 25, 2308 (2013). 